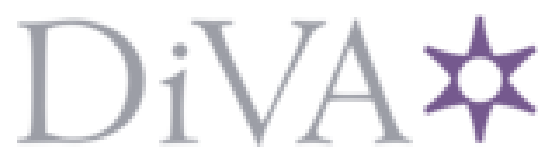

http://www.diva-portal.org

This is the published version of a paper published in Physical Review A. Atomic, Molecular, and Optical Physics.

Citation for the original published paper (version of record):

Niskanen, J., Andersson, E., Eland, J H., Linusson, P., Hedin, L. et al. (2012)

Symmetry breaking in core-valence double photoionization of $\mathrm{SO}_{2}$.

Physical Review A. Atomic, Molecular, and Optical Physics, 85(2): 023408

http://dx.doi.org/10.1103/PhysRevA.85.023408

Access to the published version may require subscription.

N.B. When citing this work, cite the original published paper.

Permanent link to this version:

http://urn.kb.se/resolve?urn=urn:nbn:se:uu:diva- 170340 


\title{
Symmetry breaking in core-valence double photoionization of $\mathrm{SO}_{2}$
}

\author{
J. Niskanen, ${ }^{1,2}$ E. Andersson, ${ }^{3}$ J. H. D. Eland, ${ }^{4,3}$ P. Linusson, ${ }^{5}$ L. Hedin,,${ }^{3}$ L. Karlsson, ${ }^{3}$ R. Feifel,,${ }^{3}$ and O. Vahtras ${ }^{1}$ \\ ${ }^{1}$ Division of Theoretical Chemistry and Biology, School of Biotechnology, Royal Institute of Technology, S-106 91 Stockholm, Sweden \\ ${ }^{2}$ Department of Physics, University of Oulu, Box 3000, FIN-90014 Oulu, Finland \\ ${ }^{3}$ Department of Physics and Astronomy, Uppsala University, Box 516, SE-751 20 Uppsala, Sweden \\ ${ }^{4}$ Department of Chemistry, Physical and Theoretical Chemistry Laboratory, Oxford University, South Parks Road, \\ Oxford OX1 3QZ, United Kingdom \\ ${ }^{5}$ Department of Physics, Stockholm University, AlbaNova University Centre, SE-106 91 Stockholm, Sweden
}

(Received 22 November 2011; published 16 February 2012)

\begin{abstract}
Core-valence double photoionization electron spectra of the $\mathrm{SO}_{2}$ molecule involving the $\mathrm{S} 2 p$ and $\mathrm{O} 1 s$ inner shells have been measured using a time-of-flight multiparticle coincidence technique. The experimental spectra are compared with quantum-chemical calculations based on density functional theory by which several core-valence dicationic states are identified. Assignments conform with a picture where the formation of a O $1 s$-valence dicationic state is associated with a physical, "pseudo-Jahn-Teller," symmetry breaking and core-hole localization. It is shown that while density functional theory gives very good transition energies in the symmetry-broken case, it gives a poor representation in the symmetry-restricted case, and an incomplete account of the Hartree-Fock localization energy.
\end{abstract}

DOI: 10.1103/PhysRevA.85.023408

PACS number(s): 33.80.Eh, 33.15.Ry, 31.15.es, 33.20.Rm

\section{INTRODUCTION}

Double ionization photoelectron spectroscopy (DIPES) provides information about the energy distribution of dicationic states of atoms and molecules and the probability for emission of two electrons upon the absorption of a single incident photon. DIPES is based upon coincidence detection of two electrons that are created in the same process, and can be obtained in an efficient way by using the time-of-flight photoelectron-photoelectron coincidence (TOF-PEPECO) spectroscopy method introduced by Eland et al. [1]. In previous works, we have made concomitant considerations of theory and computation to unravel the information content of the DIPES measurement technique $[2,3]$. We have demonstrated the versatility of the technique to explore core-valence double-hole states, both in comparison with conventional (i.e., single ionization) valence photoelectron spectra and in using designed computational protocols as helpful instruments to assign the spectra.

In this work, we extend our studies and examine the corevalence double photoionization spectra of the $\mathrm{SO}_{2}$ molecule above the $\mathrm{S} 2 p$ and $\mathrm{O} 1 s$ edges. The studied molecule plays a role in atmospheric chemistry as it constitutes one of the main pollutants in the atmosphere, emitted from both natural sources and from human activity and resulting in acid rain [4]. From a spectroscopical point of view, it is therefore interesting to characterize the electronic structure and ionization states of the $\mathrm{SO}_{2}$ molecule. The physical properties of the oxygen core-valence double ionization spectrum is of interest as it poses a symmetry dilemma similar to that of single core-hole ionization in symmetrical molecules, with the addition of a second process that also ionizes the valence levels. We find it therefore of relevance to study the symmetry-broken and symmetry-restricted core-valence double-hole electronic structures of this system and compare them with the corresponding DIPES spectrum, as it directly reflects the effects of the core hole on the valence electronic structure. The application of open-shell density functional theory makes such calculations efficient and applicable on larger scales, making it interesting to explore the performance of this theory both for the precision of energetics for core-valence hole states, and for the symmetry-breaking problem.

\section{EXPERIMENT}

The experiments were carried out at beamline U49/2-PGM2 [5] of the BESSY-II storage ring in Berlin, which was operated in single-bunch mode giving $30 \mathrm{ps} \mathrm{light} \mathrm{pulses} \mathrm{with}$ $800.5 \mathrm{~ns}$ spacing, in combination with a versatile multiparticle coincidence technique [6-8]. In the setup used, monochromatized synchrotron radiation crosses an effusive beam of target gas at one end of a magnetic bottle spectrometer $[9,10]$ formed by the divergent field of a $\sim 0.5 \mathrm{~T}$ strong, conically shaped, permanent magnet and the $\sim 10^{-3} \mathrm{~T}$ weak, uniform field of a long solenoid. Essentially all electrons created at the light-matter interaction point are guided by this arrangement of magnetic fields to the $\sim 2.2 \mathrm{~m}$ distant microchannel plate electron detector located at the other end of the flight tube. Electron flight times are measured relative to the time reference set by the ionizing light pulse and are translated, after calibration of the spectrometer against photoelectron lines and/or Auger lines of known energies from the rare gases, into electron kinetic energies. The time-to-energy conversion in the present study was established mainly by using the $\mathrm{Kr} 3 d$ photoelectron lines [11] recorded at several photon energies. The energy resolution of the electron spectrometer goes from approximately $20 \mathrm{meV}$ at the lowest kinetic energies to a nearly constant numerical resolving power of about 50 at high kinetic energies.

Because the single-bunch interpulse periods are shorter than the flight times for slow electrons, special strategies are required for unambiguous identification of the light pulse causing each ionization event [12]. In measurements performed at photon energies above the inner-shell thresholds, this can always be achieved by identification of either the innershell photoelectron peaks or associated fast Auger electron 
peaks $[8,13]$, which are of known kinetic energies and hence electron flight times.

$\mathrm{SO}_{2}$ gas was commercially obtained with a stated purity of $>99.5 \%$, and the purity of the gas was checked by on-line identification of the spectral features observed in the singleelectron spectra. Multielectron coincidence data of $\mathrm{SO}_{2}$ were recorded at the photon energies 235 and $600 \mathrm{eV}$, which are well above the thresholds for creation of the S $2 p$ and $\mathrm{O} 1 s$ core holes, respectively, along with a vacancy in a valence shell.

\section{THEORETICAL DETAILS}

The core-valence double ionization potentials (DIPs) were calculated by a recently developed, spin-restricted openshell density functional theory (DFT) [14] as the difference between the ionized-state energy and the ground-state energy. The procedure is similar to that presented in one of our recent papers on core-valence double ionization of the OCS molecule [3]. The calculations were based on a two-step relaxation process of the single core-hole state, including optimization of doubly occupied orbitals with respect to the core hole, and relaxation of the core hole itself. Doubly ionized states were then obtained by removing one electron from each valence orbital at a time. The DFT calculations were primarily performed with the Becke threeparameter Lee-Yang-Parr (B3LYP) [15] exchange-correlation functional. Other functionals [local density approximation (LDA) [16-19], Becke-88-Perdew-86 (BP86) [16-18,20-22], Perdew-Burke-Ernzerhof (PBE0) [23], Becke-Lee-Yang-Parr (BLYP) [16-20], and Coulomb-attenuated B3LYP (CAMB3LYP) [24]] were also used, producing consistent results. LDA is an exception, giving double ionization energies for the $\mathrm{O} 1 s$ case that were several $\mathrm{eV}$ different in comparison to the experimental results. In contrast, the $\mathrm{S} 2 p$ core-valence states are well produced by the LDA functional, as also found in our previous OCS study [3] (see further discussion below).

The calculations for $\mathrm{S} 2 p$ core-hole states utilized the molecular point-group symmetry $\mathrm{C}_{2 v}$, the molecule lying in the $x z$ plane. To study the localization of the $\mathrm{O} 1 s$ core hole, three different calculations were made. The first one uses localization of the $\mathrm{O} 1 s$ core hole by distorting the molecule from the $\mathrm{C}_{2 v}$ symmetry, and localizing the core hole to either one of the two $\mathrm{O}$ atoms. The second calculation uses symmetry restriction to guarantee a delocalized core hole and full $\mathrm{C}_{2 v}$ symmetry for the valence orbitals. In the third calculation, we applied the well-known $Z+1$ approximation for the core hole and obtained the DIPES spectrum with respect to the singly ionized $\mathrm{SO}_{2}$ with a localized $\mathrm{O} 1 s$ core hole. The DIP values were then obtained by adding the calculated $\mathrm{O} 1 s$ binding energy to the valence ionization potentials obtained in the $Z+1$ approximation.

The open-shell DFT calculations were performed by a local, unreleased version of the DALTON program package [25]. Following previous basis-set investigations on the $\mathrm{CS}_{2}$ and OCS molecules [2,3], the calculations were performed utilizing augmented correlation-consistent polarized core-valence triple- $\zeta$ (aug-cc-pCVTZ) basis sets of Dunning and coworkers $[26,27]$. Triplet spin coupling is employed throughout for the core-valence states, thus neglecting the splitting into singlets caused by the core-valence exchange interaction. According to previous studies, however, these splittings are expected to be small (few tenths of an eV) [2,3]. Triplets and singlets can be assumed to be statistically populated according to the number of spin sublevels.

\section{RESULTS AND DISCUSSION}

The problem of localization and symmetry breaking has been a topic of general interest for core-electron ionization. It dates back to Löwdins so-called symmetry dilemma for Hartree-Fock [28], and was studied in a number of early papers [29-35]. Bagus and Schaeffer [36] could show that a Hartree-Fock calculation of the core ionization potentials of $\mathrm{O}_{2}$ was about $15 \mathrm{eV}$ in error when full molecular symmetry was imposed, whereas the error decreased to only $1 \mathrm{eV}$ in the case of $C_{\infty v}$ symmetry. Evidence on the use of localized solutions was given by studies of other core-hole-state-related properties, such as energy gradients, vibronic coupling constants [33], electronic transition moments [37,38], angular distributions [39], and satellites [40]. The symmetry dilemma is remedied by a limited configuration-interaction calculation in the lowsymmetry point group, which retains the quasidegenerate symmetry adapted solutions. In the high symmetry, the missing relaxation can be compensated by a limited configurational expansion, with $\mathrm{O}_{2}{ }^{+}$being a prime example [34].

Correlation in the high symmetry can be related to relaxation in the low symmetry. While the symmetry breaking in homonuclear diatomic molecules does not refer to the exact wave function, but merely to an approximation of it, the problem is quite different for a triatomic molecule, such as $\mathrm{SO}_{2}$ studied here, or for any polyatomic molecules containing an element of symmetry. The symmetry can be physically broken as an effect of vibronic coupling between quasidegenerate symmetry adapted core levels. Such "pseudoJahn-Teller" effects lead to a lowering of the point group-the classical example, given by Domcke and Cederbaum [30,35], is the $\mathrm{O} 1 s^{-1} \Sigma_{u}$ and $\Sigma_{g}$ states of $\mathrm{CO}_{2}{ }^{+}$that couple over the antisymmetric stretching modes, thereby lowering the symmetry from $d_{\infty h}$ to $c_{\infty v}$ [30] (for a more recent analysis, see also Ref. [41]). Thus, even if the electronic Hamiltonian cannot break the symmetry, a vibronic Hamiltonian in a diabatic electronic representation easily displays such effects. We find it of interest to explore the symmetry breaking also for double electron ionization, as the valence structure is expected to indicate the symmetry of the potential and thus information about localization of the core hole. A relevant issue is how DFT behaves for the core-hole-state symmetry-breaking dilemma.

\section{A. The $S 2 p$ core-valence spectrum}

The $\mathrm{S} 2 p$ core-valence $\left(\mathrm{S} 2 p^{-1} v^{-1}\right)$ double ionization electron spectrum measured at the photon energy of $235 \mathrm{eV}$ is shown in Fig. 1. Several distinct structures are observed for ionization energies $>196 \mathrm{eV}$ where the onset of $\mathrm{S} 2 p^{-1} v^{-1}$ ionization is found. The first peak structure reveals a maximum at about $197 \mathrm{eV}$ and a shoulder at about $198.2 \mathrm{eV}$. This structure is followed by a double-peak structure with maxima at about 201.5 and $202.8 \mathrm{eV}$ ionization energy, respectively. Another distinct peak is observed at about $206 \mathrm{eV}$. In the higher-energy end, around $218 \mathrm{eV}$, an additional double-peak structure is found. 


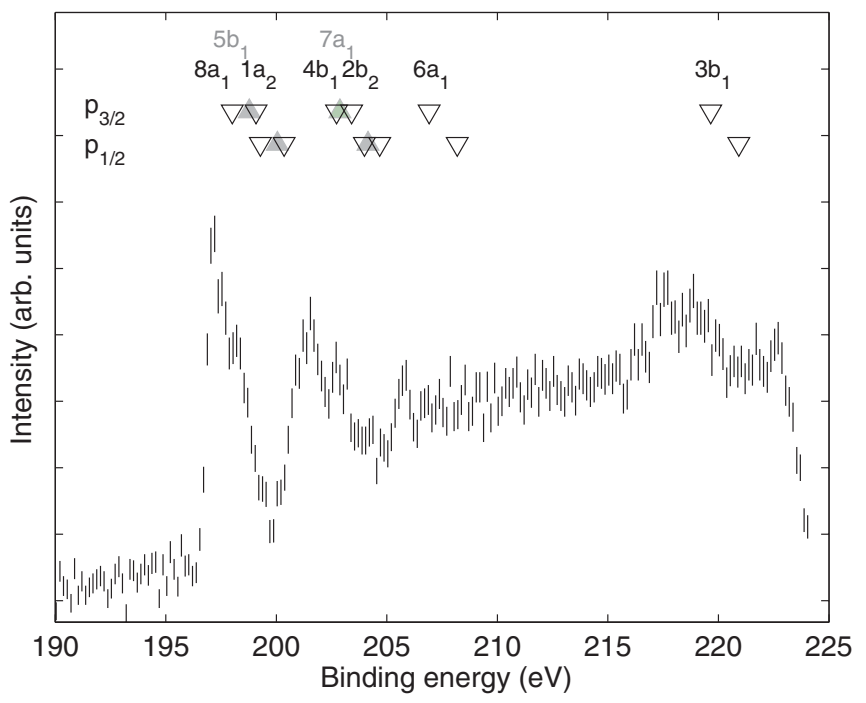

FIG. 1. (Color online) The experimental S $2 p^{-1} v^{-1}$ core-valence double ionization spectrum of the $\mathrm{SO}_{2}$ molecule recorded at $235 \mathrm{eV}$ photon energy (in our notation, $v$ stands for a valence orbital). The vertical bars present the values within one standard deviation $\sigma$. Theoretical ionization energies obtained with the B3LYP exchangecorrelation functional are presented for comparison. For clarity, the $5 b_{1}^{-1}$ and $7 a_{1}^{-1}$ states are displayed in gray. More details are given in the text.

The $\mathrm{S} 2 p^{-1} v^{-1}$ double ionization electron spectrum is expected to be characterized by the $\mathrm{S} 2 p$ spin-orbit splitting of $1.27 \mathrm{eV}[2,3]$. This splitting is clearly seen in the two energetically lowest peak structures with local intensity maxima at $\sim 197$ and $201.5 \mathrm{eV}$, respectively (cf. Fig. 1). The peak structure at approximately $205.7 \mathrm{eV}$ may also be accompanied by a second component at about $207 \mathrm{eV}$, but the latter structure is difficult to judge due to the underlying background. Again, the S $2 p$ spin-orbit splitting is clearly observed in the higher-energy structure around $218 \mathrm{eV}$.

In order to identify the spectral features observed, we incorporated in this figure S $2 p^{-1} v^{-1}$ DIP values calculated with the B3LYP exchange-correlation functional. These values are summarized in Table I where they are compared to related computational results based on the other functionals mentioned above. For the calculations, the $\mathrm{S} 2 p$ spin-orbit splitting was taken from previous studies $[2,3]$. When the perturbation matrix is set to ignore the molecular-field splitting, the $S 2 p_{3 / 2}^{-1}$ states shift $1 / 3$ of the spin-orbit splitting down in ionization energy and the $S 2 p_{1 / 2}^{-1}$ states shift $2 / 3$ of the spin-orbit splitting up in ionization energy compared to the unperturbed DIP values. The DIP values given in Table I are consistent for all chosen exchange-correlation functionals. The relative energy structure of the states remains the same, but small shifts either up or down in energy within the manifold of DIP values are encountered when comparing the results based on different functionals. We note that in agreement with our previous study [3], the LDA functional gives results similar to the other functionals.

As seen from Fig. 1, the DFT DIP values using the B3LYP functional correspond quite well to the experimental spectrum. Based on these results, the first double-peak structure in the S $2 p^{-1} v^{-1}$ electron spectrum of Fig. 1 can be assigned to almost-degenerate core-valence states involving the $8 a_{1}$, $5 b_{1}$, and $1 a_{2}$ valence orbitals, respectively. Accordingly, the double-peak structure at $201.5 \mathrm{eV}$ can be assigned to neardegenerate core-valence dicationic states involving the $7 a_{1}$, $4 b_{1}$, and $2 b_{2}$ valence orbitals, respectively. We note that in both cases, the double-peak structure is likely to be due to the spinorbit splitting, as can be seen from this comparison. The calculated core-valence dicationic states involving the $6 a_{1}$ valence orbital hole state coincide well in energy with the weaker peak structure at about $205.7 \mathrm{eV}$, suggesting that the corresponding transitions are revealed in this spectrum. Finally, core-valence states with one vacancy in the $3 b_{1}$ orbital are expected to appear just below $220 \mathrm{eV}$, slightly overestimating the DIP of the observed lines. From a computational point of view, as can be learned from this comparison, the experimental $\mathrm{S} 2 p$ core-valence spectrum of $\mathrm{SO}_{2}$ can be modeled and understood very well with the open-shell DFT approach. Furthermore, we note that all exchange-correlation functionals employed here give results which are in agreement with each other.

\section{B. The $O 1 s$ core-valence spectrum}

The $\mathrm{O} 1 s$ core-valence $\left(\mathrm{O} 1 s^{-1} v^{-1}\right)$ double ionization electron spectrum of $\mathrm{SO}_{2}$ measured at the photon energy of $600 \mathrm{eV}$ is shown in Fig. 2. Akin to the core-valence spectrum measured in the vicinity of the $\mathrm{S} 2 p$ core hole, several distinct structures

TABLE I. The DFT S $2 p^{-1} v^{-1}$ double ionization energies (in eV) obtained using a selected choice of exchange-correlation functionals (in our notation, $v$ stands for a valence orbital). For reference, the experimental S $2 p_{3 / 2}$ single ionization energy is at $\sim 174.8 \mathrm{eV}$ (cf. Ref. [42]).

\begin{tabular}{|c|c|c|c|c|c|c|c|c|c|c|c|c|}
\hline \multirow[b]{2}{*}{ Configuration } & \multicolumn{2}{|c|}{ B3LYP } & \multicolumn{2}{|c|}{ BP86 } & \multicolumn{2}{|c|}{ PBE0 } & \multicolumn{2}{|c|}{ CAM-B3LYP } & \multicolumn{2}{|c|}{ BLYP } & \multicolumn{2}{|c|}{ LDA } \\
\hline & $p_{3 / 2}$ & $p_{1 / 2}$ & $p_{3 / 2}$ & $p_{1 / 2}$ & $p_{3 / 2}$ & $p_{1 / 2}$ & $p_{3 / 2}$ & $p_{1 / 2}$ & $p_{3 / 2}$ & $p_{1 / 2}$ & $p_{3 / 2}$ & $p_{1 / 2}$ \\
\hline S $2 p^{-1}$ & 174.59 & 175.86 & 173.85 & 175.12 & 174.39 & 175.66 & 174.80 & 176.07 & 173.84 & 175.11 & 174.33 & 175.60 \\
\hline S $2 p^{-1} 8 a_{1}^{-1}$ & 198.01 & 199.28 & 197.09 & 198.36 & 197.87 & 199.14 & 198.50 & 199.77 & 196.90 & 198.17 & 197.71 & 198.98 \\
\hline S $2 p^{-1} 7 a_{1}^{-1}$ & 202.88 & 204.15 & 201.70 & 202.97 & 202.91 & 204.18 & 203.57 & 204.84 & 201.44 & 202.71 & 202.34 & 203.61 \\
\hline S $2 p^{-1} 6 a_{1}^{-1}$ & 206.92 & 208.19 & 205.52 & 206.79 & 207.01 & 208.28 & 207.64 & 208.91 & 205.26 & 206.53 & 205.91 & 207.18 \\
\hline S $2 p^{-1} 2 b_{2}^{-1}$ & 203.40 & 204.67 & 202.60 & 203.87 & 203.33 & 204.60 & 203.97 & 205.24 & 202.35 & 203.62 & 203.38 & 204.65 \\
\hline S $2 p^{-1} 5 b_{1}^{-1}$ & 198.78 & 200.05 & 197.92 & 199.19 & 198.67 & 199.94 & 199.28 & 200.55 & 197.71 & 198.98 & 198.62 & 199.89 \\
\hline S $2 p^{-1} 4 b_{1}^{-1}$ & 202.72 & 203.99 & 201.62 & 202.89 & 202.73 & 204.00 & 203.34 & 204.61 & 201.37 & 202.64 & 202.19 & 203.46 \\
\hline S $2 p^{-1} 3 b_{1}^{-1}$ & 219.65 & 220.92 & 217.94 & 219.21 & 219.87 & 221.14 & 220.37 & 221.64 & 217.65 & 218.92 & 218.36 & 219.63 \\
\hline S $2 p^{-1} 1 a_{2}^{-1}$ & 199.08 & 200.35 & 198.62 & 199.89 & 198.93 & 200.20 & 199.40 & 200.67 & 198.38 & 199.65 & 199.40 & 200.67 \\
\hline
\end{tabular}




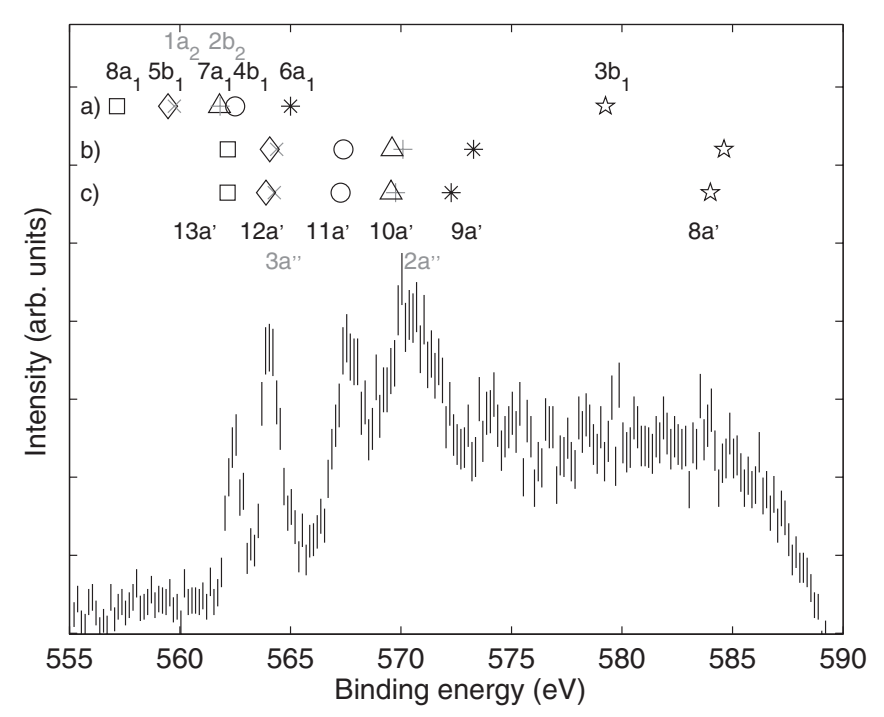

FIG. 2. The experimental O $1 s^{-1} v^{-1}$ core-valence double ionization spectrum of the $\mathrm{SO}_{2}$ molecule recorded at a photon energy of $600 \mathrm{eV}$ (in our notation, $v$ stands for a valence orbital). The vertical bars present the values within one standard deviation $\sigma$. Theoretical ionization energies obtained with the B3LYP exchange-correlation functional in the (a) symmetry-restricted, (b) $Z+1$ approximation, and (c) symmetry-broken models are presented. The dicationic Hamiltonian eigenstates are assigned with individual markers to visualize their correspondence in the different models (more details are given in the text).

are revealed. As can be seen, the $\mathrm{O} 1 s^{-1} v^{-1}$ onset is found to be at $\sim 562 \mathrm{eV}$. The first peak located at approximately $562.3 \mathrm{eV}$ is accompanied by an even more intense feature at about $564 \mathrm{eV}$ ionization energy. This peak structure is followed by other strong structures with peak maxima at about 567.5 and $570 \mathrm{eV}$, respectively. In this higher energy region, the background is increasing and definite statements on the actual intensities are difficult. Above the apparently strongest peak structure at about $570 \mathrm{eV}$, an additional weaker feature at about $574 \mathrm{eV}$ is discernable in the spectrum.

In order to assign the structures observed, we incorporated in this figure the $\mathrm{O} 1 s^{-1} v^{-1}$ DIP values calculated with the B3LYP exchange-correlation functional in three different models: (a) delocalized core hole, (b) $Z+1$ approximation, and (c) localized core hole. The localized core values are summarized in Table II, together with results based on other functionals mentioned above. Localization of the core hole implies a breakdown of the initial symmetry of the molecule as the core-hole site appears almost charged as $Z+1$ to the rest of the molecule, when neglecting the comparatively small exchange effects. The actual symmetry relevant for the molecular valence orbitals is thus represented by the molecular symmetry group $\mathrm{C}_{s}$, where only the molecular plane ( $x z$ plane) acts as the mirror plane and the valence orbitals are labeled accordingly.

The calculated DIP values in Table II are quite consistent, except for the LDA functional. This finding is in agreement with our previous study on core-valence double ionization of the OCS molecule [3]. In comparing absolute DIP values obtained for different functionals, small variations are seen, akin to our results obtained in the vicinity of the $\mathrm{S} 2 p$ core holes (cf. Table I), but the relative energy structure of the states is very similar.

Based on these numerical results, the lowest peak structure in the $\mathrm{O} 1 s^{-1} v^{-1}$ core-valence spectrum can be assigned to a dicationic state involving the $13 a^{\prime}$ valence orbital, and the structure at about $564 \mathrm{eV}$ can be assigned to dicationic states involving the $12 a^{\prime}$ and $3 a^{\prime \prime}$ valence orbitals. The higher intensity of the latter peak structure can qualitatively be explained by a double degeneracy, even though the dynamics and cross section of core-valence double photoionization was not modeled in the present work. The spectral feature at about $567.5 \mathrm{eV}$ can be assigned to a dicationic state involving the $11 a^{\prime}$ valence orbital vacancy, and the peak at about $570 \mathrm{eV}$ is assigned to dicationic states involving the $10 a^{\prime}$ and $2 a^{\prime \prime}$ valence holes. Akin to the two lowest features in the spectrum, the intensities of these two peaks can be explained qualitatively by degeneracy. The calculations suggest that the weak peak structure at $574 \mathrm{eV}$ is due to core-valence double ionization leading to states of $\mathrm{O} 1 s^{-1} 9 a^{-1}$ configuration. Finally, the $\mathrm{O} 1 s^{-1} 8 a^{\prime-1}$ states are suggested to be present in the higher-energy region above $582 \mathrm{eV}$ ionization energy, but left experimentally undetected.

The $\mathrm{C}_{2 v}$ symmetry point group exhibits only nondegenerate irreducible representations. Therefore, all near-degeneracy (or "closeness" in energy) of dicationic core-valence states takes place by "chance," rather than due to molecular symmetry.

TABLE II. The DFT O $1 s^{-1} v^{-1}$ double ionization energies (in eV) obtained using the selected exchange-correlation functionals and the symmetry-broken, localized $\mathrm{O} 1 s$ core-hole model (in our notation, $v$ stands for a valence orbital). For each state, the closest corresponding configuration in the $\mathrm{C}_{2 v}$ symmetry is given in the parenthesis. For reference, the experimental $\mathrm{O} 1 s$ single ionization energy is $539.6 \mathrm{eV}$ (cf. Ref. [42]).

\begin{tabular}{|c|c|c|c|c|c|c|c|}
\hline Config. $\mathrm{C}_{s}$ & Config. $\mathrm{C}_{2 v}$ & B3LYP & BP86 & PBE0 & CAM-B3LYP & BLYP & LDA \\
\hline $\mathrm{O} 1 s^{-1}$ & & 539.45 & 539.49 & 539.06 & 539.64 & 539.45 & 534.92 \\
\hline O $1 s^{-1} 13 a^{\prime-1}$ & $\left(\mathrm{O} 1 s^{-1} 8 a_{1}^{-1}\right)$ & 562.15 & 562.19 & 561.81 & 562.55 & 561.97 & 557.79 \\
\hline $\mathrm{O} 1 s^{-1} 12 a^{\prime-1}$ & $\left(\mathrm{O} 1 s^{-1} 5 b_{1}^{-1}\right)$ & 563.89 & 563.81 & 563.62 & 564.35 & 563.55 & 559.45 \\
\hline $\mathrm{O} 1 s^{-1} 11 a^{\prime-1}$ & $\left(\mathrm{O} 1 s^{-1} 4 b_{1}^{-1}\right)$ & 567.27 & 566.91 & 567.09 & 567.85 & 566.63 & 562.41 \\
\hline O $1 s^{-1} 10 a^{\prime-1}$ & $\left(\mathrm{O} 1 s^{-1} 7 a_{1}^{-1}\right)$ & 569.55 & 569.27 & 569.24 & 570.03 & 569.05 & 565.05 \\
\hline O $1 s^{-1} 9 a^{\prime-1}$ & $\left(\mathrm{O} 1 s^{-1} 6 a_{1}^{-1}\right)$ & 572.27 & 571.88 & 572.09 & 572.83 & 571.61 & 567.49 \\
\hline O $1 s^{-1} 8 a^{\prime-1}$ & $\left(\mathrm{O} 1 s^{-1} 3 b_{1}^{-1}\right)$ & 584.00 & 583.43 & 583.96 & 584.51 & 583.09 & 578.78 \\
\hline $\mathrm{O} 1 s^{-1} 3 a^{\prime \prime-1}$ & $\left(\mathrm{O} 1 s^{-1} 1 a_{2}^{-1}\right)$ & 564.27 & 564.50 & 563.92 & 564.58 & 564.23 & 560.25 \\
\hline $\mathrm{O} 1 s^{-1} 2 a^{\prime \prime-1}$ & $\left(\mathrm{O} 1 s^{-1} 2 b_{2}^{-1}\right)$ & 569.75 & 569.65 & 569.39 & 570.19 & 569.45 & 565.46 \\
\hline
\end{tabular}


However, according to Fig. 1, there is triple near degeneracy in the two lowest pairs of S $2 p^{-1} v^{-1}$ core-valence double ionization lines, which is partially removed in the $\mathrm{O} 1 s^{-1} v^{-1}$ spectrum. First, the near degeneracy of $8 a_{1}, 5 b_{1}$, and $1 a_{2}$ valence states in the $\mathrm{S} 2 p^{-1} v^{-1}$ spectrum of Fig. 1 is found to split to two components in the $\mathrm{O} 1 s^{-1} v^{-1}$ spectrum. This splitting is evident in all cases of symmetry-restricted, $Z+1$ approximation and localized core models, and results in one line for $13 a^{\prime-1}$ and one line for $12 a^{\prime-1}$ and $3 a^{\prime \prime-1}$ valence vacancies. More interestingly, the almost-degenerate $7 a_{1}^{-1}$, $4 b_{1}^{-1}$, and $2 b_{2}^{-1}$ valence states in the spectrum of Fig. 1 split in two in the spectra of Fig. 2, but only when the localized $\mathrm{O} 1 s$ core-hole model is used $(Z+1$ and localized case). This removal of degeneracy is seen as a line for $11 a^{\prime-1}$ and a broader one for $10 a^{\prime-1}$ and $2 a^{\prime \prime-1}$. We also point out that the whole $\mathrm{O} 1 s^{-1} v^{-1}$ spectrum is shifted to $5 \mathrm{eV}$ lower energies when the $\mathrm{C}_{2 v}$ symmetry (and delocalization) requirement is set.

The symmetry-restricted model (a) in Fig. 2 gives DIP values and relative line structure that are not in agreement with the experimental spectrum or the DIP values obtained by the other models (b) and (c). The latter two give consistent results, which support the conclusion of a well-localized core hole. Moreover, Fig. 2 shows that the localized core-hole model (c) and $Z+1$ approximation (b) are in very good agreement with the experiment, making an identification of the spectral features possible. The good agreement with the experimental spectrum is also a strong argument for the core-hole localization. Symmetry breaking is also directly evidenced by a geometry optimization of the core-hole states, which indicate an asymmetric stretch displacement to $\mathrm{S}-\mathrm{O} *=+0.042 \AA$ and $\mathrm{S}-\mathrm{O}=-0.042 \AA$ ( $*$ marks the ionized core atom). Thus indeed the generation of core-valence holes is followed by physical symmetry breaking and hole localization. It is interesting to note that DFT, shown above to generate a similar good quality of transition energies in the symmetry-broken case as in the normal case [43-45], behaves rather poorly for the symmetry-restricted case: For the symmetry-restricted optimized core-hole state, the ionization potential (IP) is $533.74 \mathrm{eV}$ (with B3LYP at ground-state geometry), while the corresponding localized optimized energy is $539.42 \mathrm{eV}$. The corresponding values for Hartree-Fock are 551.22 and $539.17 \mathrm{eV}$, respectively (experiment: $539.7 \mathrm{eV}$ [42]). We note that the DFT "orbital energy" is approximately $-522 \mathrm{eV}$, thus on the "wrong side" in the Hartree-Fock sense of the relaxed IP. This is the normal case and something that refers to strong core-electron self-interaction, which is present in DFT but which is correctly nullified in Hartree-Fock. For nonsymmetry molecules (and apparently here for symmetry-breaking cases), Kohn-Sham orbital reoptimization in the opened core-hole potential [45] removes the problem and generates IPs typically within an $\mathrm{eV}$ of experiment. It is interesting that DFT improves the Hartree-Fock error in absolute magnitude (roughly by a factor of 2), but approaches the correct value still from "the wrong side". The lack of static, near-degenerate correlation, which certainly is important for the core-hole symmetry dilemma [34], probably contributes to the deficiency of the DFT IP in the symmetry-restricted case. Thus, at least in part, DFT mimicks Hartree-Fock for symmetry breaking, which may not be surprising in that DFT essentially is a short-range dynamical correlation theory, and does not account for long-range strong correlation effects. However, the analysis is aggravated by the presence of the self-interaction error in DFT.

\section{CONCLUSIONS}

We presented the $\mathrm{S} 2 p^{-1} v^{-1}$ and $\mathrm{O} 1 s^{-1} v^{-1}$ core-valence double ionization electron spectra of the $\mathrm{SO}_{2}$ molecule. The experimental spectra were compared with DFT calculations and many core-valence dicationic states were identified. The energies of the spectral features observed in the $\mathrm{S} 2 p^{-1} v^{-1}$ spectrum were reproduced well by all functionals applied. In the case of the $\mathrm{O} 1 s^{-1} v^{-1}$ spectrum, the double ionization potentials obtained by the LDA exchange-correlation functional were underestimating the double ionization energies for the $\mathrm{O} 1 s^{-1} v^{-1}$ states by $4-5 \mathrm{eV}$, which can be explained by the large self-interaction error for core electrons in that functional.

Symmetry breaking due to the localization of the $\mathrm{O}$ $1 s$ core hole affecting the molecular valence orbitals was observed as a removal of a near degeneracy observed in the $\mathrm{S} 2 p^{-1}\left(7 a_{1}, 4 b_{1}, 2 b_{2}\right)^{-1}$ core-valence spectrum. To study the core-hole localization, symmetry-broken, symmetryrestricted, and $Z+1$ approximation models were used. The symmetry-broken $\mathrm{O} 1 \mathrm{~s}$ spectrum showed almost perfect agreement with the experiment, whereas the symmetryrestricted solution failed in describing the experiment in comparable accuracy, resulting also in a shift of $5 \mathrm{eV}$ in the dicationic state energies. On the other hand, the coarse energy splitting of states remained largely the same in the models, indicating that symmetry-induced relaxation is common to all levels. Geometry optimization also indicates physical symmetry breaking by a displacement along the antisymmetric stretch. We find that DFT accounts for roughly half of the magnitude of the Hartree-Fock localization energy. DFT lacks important (near-degenerate) correlation that corresponds to relaxation contributions in the symmetry-broken case, but suffers also from self-interaction, which aggravates the analysis. In the symmetry-broken case, DFT gives excellent energetics.

The spin-restricted DFT was found to perform very well in comparison with earlier studies obtained with the highly correlated $a b$ initio calculations. As DFT dynamically correlate all electrons, there is no need for energy corrections assign the DIPES spectrum. The accuracy of energetics obtained by DFT, and the good system-size scaling properties of the theory have important ramifications for analyzing larger systems.

\section{ACKNOWLEDGMENTS}

This work has been financially supported by the Swedish Research Council (V.R.), the Göran Gustafsson Foundation (U.U. and K.T.H.), and the Knut and Alice Wallenberg Foundation, Sweden. This work was also supported by the European Community - Research Infrastructure Action under the FP6 "Structuring the European Research Area" Programme (through the Integrated Infrastructure Initiative "Integrating Activity on Synchrotron and Free Electron Laser Science," Contract No. R II 3-CT-2004-506008). 
[1] J. H. D. Eland, O. Vieuxmaire, T. Kinugawa, P. Lablanquie, R. I. Hall, and F. Penent, Phys. Rev. Lett. 90, 053003 (2003).

[2] E. Andersson, J. Niskanen, L. Hedin, J. H. D. Eland, P. Linusson, L. Karlsson, J.-E. Rubensson, V. Carravetta, H. Ågren, and R. Feifel, J. Chem. Phys. 133, 094305 (2010).

[3] J. Niskanen, V. Carravetta, O. Vahtras, H. Ågren, H. Aksela, E. Andersson, L. Hedin, P. Linusson, J. H. D. Eland, L. Karlsson, J.-E. Rubensson, and R. Feifel, Phys. Rev. A 82, 043436 (2010).

[4] J. H. Seinfeld and S. N. Pandis, Atmospheric Chemistry and Physics: From Air Pollution to Climate Change, 2nd ed. (Wiley, New Jersey, 2006).

[5] D. R. Batchelor, R. Follath, and D. Schmeisser, Nucl. Instrum. Methods A 467, 470 (2001).

[6] J. H. D. Eland and R. Feifel, Chem. Phys. 327, 85 (2006).

[7] R. Feifel, J. H. D. Eland, L. Storchi, and F. Tarantelli, J. Chem. Phys. 125, 194318 (2006).

[8] E. Andersson, M. Stenrup, J. H. D. Eland, L. Hedin, M. Berglund, L. Karlsson, Å. Larson, H. Ågren, J.-E. Rubensson, and R. Feifel, Phys. Rev. A 78, 023409 (2008).

[9] J. H. D. Eland, O. Vieuxmaire, T. Kinugawa, P. Lablanquie, R. I. Hall, and F. Penent, Phys. Rev. Lett. 90, 053003 (2003).

[10] P. Kruit and F. H. Read, J. Phys. E 16, 313 (1983).

[11] Z.-S. Yuan, L.-F. Zhu, X.-J. Liu, W.-B. Li, H.-D. Cheng, J.-M. Sun, and K.-Z. Xu, Phys. Rev. A 71, 064701 (2005).

[12] S. Plogmaker, P. Linusson, J. H. D. Eland, E. M. J. Johansson, H. Rensmo, R. Feifel, and H. Siegbahn (unpublished).

[13] F. Penent, J. Palaudoux, P. Lablanquie, L. Andric, R. Feifel, and J. H. D. Eland, Phys. Rev. Lett. 95, 083002 (2005).

[14] Z. Rinkevicius, I. Tunell, P. Sałek, O. Vahtras, and H. Ågren, J. Chem. Phys. 119, 34 (2003).

[15] A. D. Becke, J. Chem. Phys. 98, 5648 (1993).

[16] P. Hohenberg and W. Kohn, Phys. Rev. 136, B864 (1964).

[17] W. Kohn and L. J. Sham, Phys. Rev. 140, A1133 (1965).

[18] J. C. Slater, Quantum Theory of Molecular and Solids (McGrawHill, New York, 1974), Vol. 4.

[19] S. H. Vosko, L. Wilk, and M. Nusair, Can. J. Phys. 58, 1200 (1980).

[20] A. D. Becke, Phys. Rev. A 38, 3098 (1988).

[21] J. P. Perdew and A. Zunger, Phys. Rev. B 23, 5048 (1981).

[22] J. P. Perdew, Phys. Rev. B 33, 8822 (1986).

[23] J. P. Perdew, K. Burke, and M. Ernzerhof, Phys. Rev. Lett. 77, 3865 (1996).

[24] T. Yanai, D. P. Tew, and N. C. Handy, Chem. Phys. Lett. 393, 51 (2004).
[25] C. Angeli, K. L. Bak, V. Bakken, O. Christiansen, R. Cimiraglia, S. Coriani, P. Dahle, E. K. Dalskov, T. Enevoldsen, B. Fernandez, C. Hättig, K. Hald, A. Halkier, H. Heiberg, T. Helgaker, H. Hettema, H. J. Aa. Jensen, D. Jonsson, P. Jørgensen, S. Kirpekar, W. Klopper, R. Kobayashi, H. Koch, A. Ligabue, O. B. Lutnæs, K. V. Mikkelsen, P. Norman, J. Olsen, M. J. Packer, T. B. Pedersen, Z. Rinkevicius, E. Rudberg, T. A. Ruden, K. Ruud, P. Salek, A. Sanchez de Meras, T. Saue, S. P. A. Sauer, B. Schimmelpfennig, K. O. Sylvester-Hvid, P. R. Taylor, O. Vahtras, D. J. Wilson, and H. Ågren, computer code DALTON 2.0 (http://www.daltonprogram.org, 2005).

[26] T. H. Dunning Jr., J. Chem. Phys. 90, 1007 (1989).

[27] D. E. Woon and T. H. Dunning Jr., J. Chem. Phys. 98, 1358 (1993).

[28] P.-O. Löwdin, Rev. Mod. Phys. 35, 496 (1963).

[29] H. T. Jonkman, G. A. van der Welde, and W. C. Nieuwport, Chem. Phys. Lett. 25, 62 (1974).

[30] W. Domcke and L. S. Cederbaum, Chem. Phys. 25, 189 (1976).

[31] W. Butscher, R. J. Buenker, and S. D. Peyerimhoff, Chem. Phys. Lett. 52, 449 (1977).

[32] S. Canuto, O. Goscinski, and M. Zerner, Chem. Phys. Lett. 68, 232 (1979).

[33] J. Müller, H. Ågren, and O. Goscinski, Chem. Phys. 38, 349 (1979).

[34] H. Ågren, P. S. Bagus, and B. O. Roos, Chem. Phys. Lett. 82, 505 (1981).

[35] L. S. Cederbaum and W. Domcke, J. Chem. Phys. 66, 5084 (1977).

[36] P. S. Bagus and H. F. Schaefer, J. Chem. Phys. 56, 224 (1972).

[37] H. Ågren and J. Nordgren, Theor. Chim. Acta 58, 111 (1981).

[38] H. Ågren, A. Cesar, and C.-M. Liegener, Adv. Quantum Chem. 23, 1 (1992).

[39] D. Dill, S. Wallace, J. Siegel, and J. L. Dehmer, Phys. Rev. Lett. 41, 1230 (1978).

[40] R. Arneberg, H. Ågren, P. A.. Malmquist, and S. Svensson, Chem. Phys. Lett. 92, 125 (1982).

[41] A. Cesar, F. Gel'mukhanov, Y. Luo, H. Ågren, P. Skytt, P. Glans, J.-H. Guo, K. Gunnelin, and J. Nordgren, J. Chem. Phys. 106, 3439 (1997).

[42] K. Siegbahn, C. Nordling, G. Johansson, J. Hedman, P. F. Hedén, K. Hamrin, U. Gelius, T. Bergmark, L. O. Werme, R. Manne, and Y. Baer, ESCA Applied to Free Molecules (North-Holland, Amsterdam, 1969).

[43] D. P. Chong, Chem. Phys. Lett. 232, 486 (1995).

[44] L. Triguero, L. G. M. Pettersson, and H. Agren, Phys. Rev. B 58, 8097 (1998).

[45] L. Triguero, O. Plashkevych, L. G. M. Pettersson, and H. Ågren, J. Electr. Spectrosc. Rel. Phenom. 104, 195 (1999). 